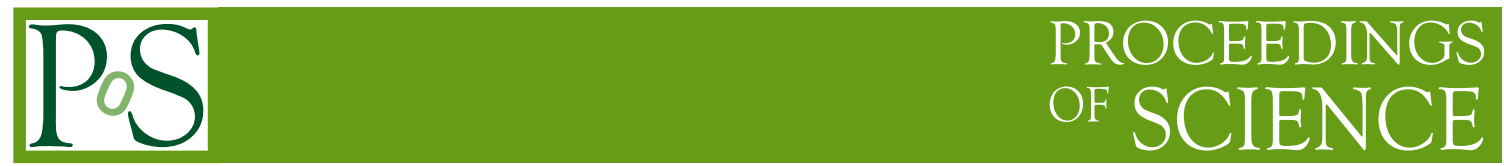

\title{
Neutrino Oscillations in the NOvA Experiment
}

\author{
Alec Habig ${ }^{* \dagger}$ \\ University of Minnesota Duluth \\ E-mail: ahabig@umn.edu

\section{Maury Goodman} \\ Argonne National Lab \\ E-mail: maury.goodmaneanl.gov
}

\section{For the NOvA Collaboration}

Analysis of an exposure of $6 \times 10^{20}$ protons on target of the NuMI muon neutrino beam have produced precision measurements of both disappearance and appearance oscillations, including the first hints that muon to tau neutrino mixing is not maximal.

35th International Cosmic Ray Conference - ICRC2017

10-20 July, 2017

Bexco, Busan, Korea

${ }^{*}$ Speaker.

${ }^{\dagger}$ Supported by NSF RUI \#1607381 


\section{Overview}

The NOvA experiment [1] is a long-baseline neutrino oscillation experiment which uses two similar detectors $810 \mathrm{~km}$ apart to study neutrino oscillations at an angle of $14.6 \mathrm{mrad}\left(0.837^{\circ}\right)$ from the focusing axis of Fermilab's NuMI $[2,3]$ neutrino beam. This configuration results in a narrowband beam of $v_{\mu}$ peaked between $1-3 \mathrm{GeV}$, with a L/E right on the first oscillation minimum in the "atmospheric" sector. The off-axis configuration not only reduces uncertainty in the incoming neutrino energy, but also reduces the background of higher-energy neutrinos producing neutral current interactions that might reconstruct as electron neutrinos in the region of interest. However, this does result in a far lower intensity than is available on-axis, mitigated by a large far detector and the fact that the NuMI beam is now routinely operating at its $700 \mathrm{~kW}$ design rating. This poster describes the neutrino oscillation analyses of the first $6.05 \times 10^{20}$ protons-on-target ("pot") of fulldetector equivalent exposure of the NOvA detectors in the NuMI beam, as described in $[4,5]$.

\section{Goals}

NOvA's primary goal is to observe $v_{e}$ appearance in the predominantly $v_{\mu}$ NuMI beam by comparing the neutrino spectra observed at the near and far detectors. The mixing angle associated with this oscillation $\left(\theta_{13}\right)$ is now well measured and is comparatively large [6]: thus interesting sub-dominant effects such as the phase of $\mathrm{CP}$ violation in the neutrino sector $\delta_{C P}$ and the hierarchy of the neutrino mass states could be detected, as well as determining the octant of $\theta_{23}$. The best measurements of these parameters will be obtained by comparing the oscillations observed in a neutrino beam with those seen in an anti-neutrino beam: NuMI has been running as an anti- $v_{\mu}$ beam since 2016, although analysis of that data is ongoing so this poster uses only $v_{\mu}$ beam data. Measuring the degree of $v_{\mu}$ disappearance can tell if $\theta_{23}$ is maximal or not and factors into the uncertainties in the $v_{e}$ related parameters.

The $\mathrm{CP}$ and mass hierarchy effects on oscillations are dependent on the number of electrons in the path of the beam. NOvA has the longest baseline of any accelerator neutrino experiment, thus increasing sensitivity to part of the range of possible parameters. If neutrino mass states are arranged in a "Normal Hierarchy" where the solar doublet of mass eigenstates is larger than the third state, there would be an enhancement of the $v_{\mu} \rightarrow v_{e}$ appearance caused by coherent forward scattering of $v-e$ on electrons in the earth. The "Inverted Hierarchy" would behave in the opposite fashion. Comparisons to the similar effects seen in the T2K experiment in Japan [7] will allow for more sensitivity than either experiment alone, as T2K and NOvA have beams of different energies and baselines, and possess different systematic errors given the complementary natures of their detectors.

\section{The Detectors}

The NOvA detectors are constructed to be as similar as possible aside from size: the far detector is $14 \mathrm{kt}$ to maximize statistics at the northernmost point of the baseline in Ash River, MN; while the near detector is close enough to the beam at Fermilab to see a far greater flux, so only needs to be $300 \mathrm{t}$. Both are constructed out of extruded PVC cells $3.9 \times 6.6 \mathrm{~cm}$ in cross section 
filled with liquid scintillator, $15.5 \mathrm{~m}$ long at the far detector and $3.8 \mathrm{~m}$ long at the near detector. Light is piped out of the cell by a length of wavelength shifting fiber looped to the end of each cell and back, allowing avalanche photo-diodes to digitize the light and record when a charged particle has traversed a cell and how much light it created. The cells alternate between vertically and horizontally oriented to allow for a stereo readout. Scintillator itself composes $62 \%$ of the mass of the detector, and the low- $\mathrm{Z}$ nature of all the components allow for a long radiation length of $35.8 \mathrm{~cm}$. This allows electromagnetic showers from potential neutral current background to be well resolved. Fig. 1 shows how the pattern of scintillator light distributed across the detector can be used to tell apart different kinds of neutrino interactions.

Unlike other neutrino experiments, the NOvA far detector is on the surface with minimal overburden. Its large size means that the cosmic ray rate is $148 \mathrm{kHz}$, producing a volume of more than $1 \mathrm{~GB} / \mathrm{s}$ of data. To sort out the rare neutrino interactions, the time of the $10 \mu \mathrm{s}$ beam spill at Fermilab is sent to the far detector and a window of data surrounding that time is saved. This reduces the cosmic ray background by a factor of $10^{5}$, as in that short time few cosmic rays are coincident in time. These remaining cosmics can be identified as entering events and discarded, while neutrino interactions start well inside the detector volume, for an additional reduction in cosmic background of $10^{7}\left(10^{8}\right.$ for $v_{e}$ candidates), for a total reduction of $>10^{12}$. For non-beam physics, a trigger farm examines the data in real time to save interesting data [8]. For example, a burst of neutrinos from a core-collapse supernova in our galaxy would create an increased rate of $10 \mathrm{MeV}$ positrons via inverse beta decay, if we can trigger on this signature [9]. Given the high rate and good spatial resolution, cosmic ray physics can be also done with both near and far detectors [10].

The NuMI beam was operational as the far detector was constructed and commissioned, with physics data being recorded from February 6, 2014. The modular nature of the detector allowed collecting of neutrino data using the partially built part of the detector. Both detectors were complete in August 2014 with the final commissioning at the far detector finished in November 2014. Scaling the exposure used in this poster by far detector mass, an equivalent of $6.05 \times 10^{20}$ pot on a full $14 \mathrm{kt}$ was collected before May 2, 2016.

\section{Muon Neutrino Disappearance}

The NuMI neutrino beam is observed at Fermilab in the near detector before neutrinos have had a chance to oscillate. This establishes the initial spectrum and composition of the beam. Comparing this to the spectrum and composition of the beam $810 \mathrm{~km}$ away at the far detector allows the estimation of what values of the neutrino oscillation parameters must be operating on the neutrinos during their journey northwards to change the near to the far spectrum. Assuming no oscillations, the beam at the near (and far) detector is composed of $97.5 \%(97.8 \%) v_{\mu}, 1.8 \%(1.6 \%) \bar{v}_{\mu}$ and $0.7 \%(0.6 \%) v_{e}+\bar{v}_{e}$ between $1-3 \mathrm{GeV}$. The slight differences are caused by the small differences in kinematic phase space subtended by the two detectors.

As seen in Fig. 1, the different flavors of neutrinos can be easily discerned by the topology of the charged leptons produced in a charge current interaction. However, neutral current interactions are the same regardless of the flavor of the incoming neutrino. Thus, neutral current interactions which are mis-reconstructed as a charged current interaction form the dominant background to a 


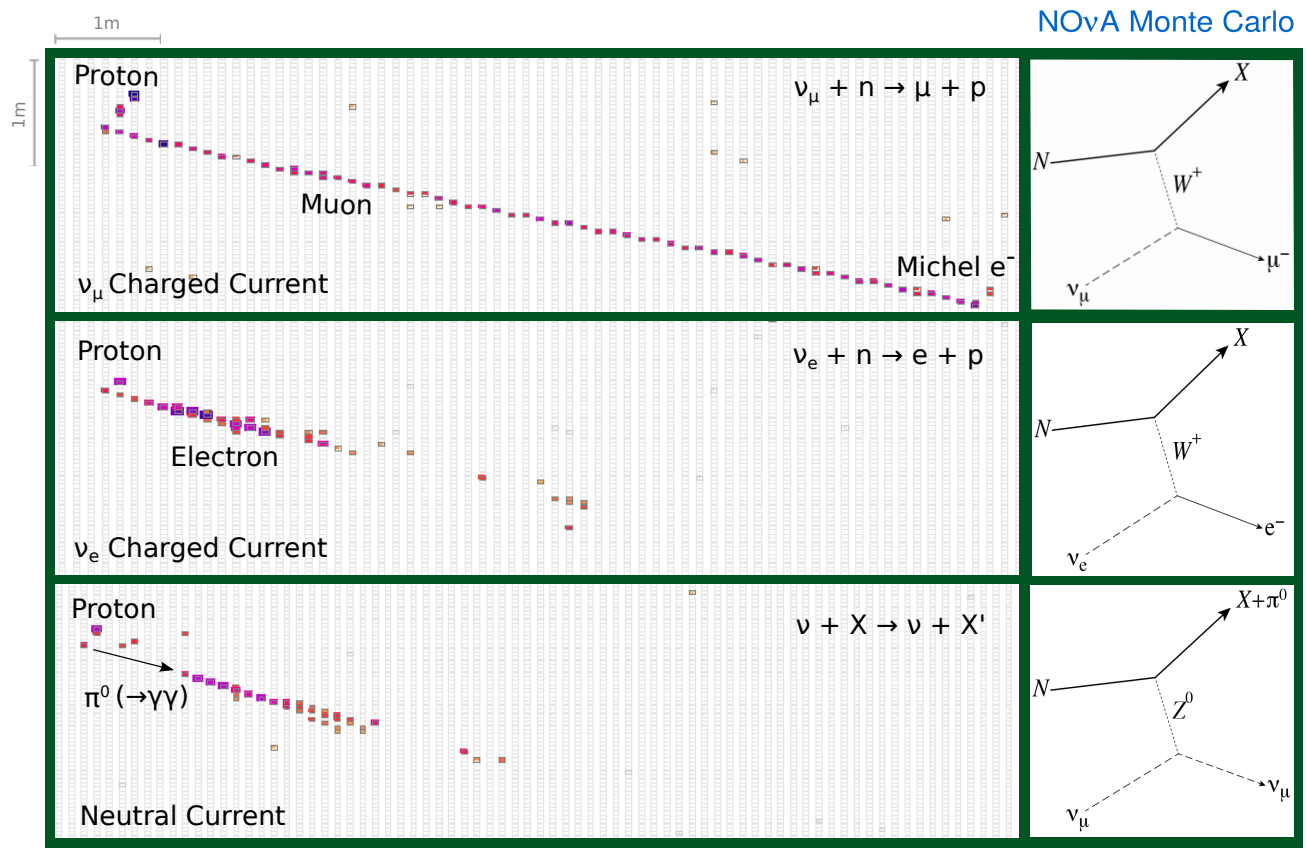

Figure 1: Event displays of simulated neutrino interactions in NOvA. Three different types of neutrino interactions are shown. Each rectangular "pixel" in the displays on the left corresponds to an end-on view of one cell in which passage of a charged particle created some scintillator light. The Feynman diagram of each weak interaction is on the right. In the top interaction, a $v_{\mu}$ turns a neutron into a proton which recoils (the short stubby track), with the outgoing lepton producing a long straight track indicative of a muon. In the second interaction, a $v_{e}$ does produces an electron, which initiates a shorter, fuzzier electromagnetic shower. The third interaction is a neutral current interaction where the neutrino recoils from a proton, producing a neutral pion which rapidly decays into two gamma rays. Note the gap between the interaction vertex and the start of the electromagnetic shower, corresponding to the radiation length of a gamma in the light hydrocarbons which compose the NOvA detector.

flavor-based analysis. While the off-axis nature of the experiment greatly reduces the number of neutral current interactions which produce something that looks like a $\sim 2 \mathrm{GeV}$ charged current interaction, each neutrino candidate is examined by a collection of tracking algorithms (see [4] for details), with events matching $v_{\mu}$ charged current interactions being kinematically reconstructed and plotted as a function of energy as shown in the left of Fig. 2. Performing the same reconstruction on Monte Carlo data shows it to be $62 \%$ efficient at retaining true $v_{\mu}$ charged current interactions, with an overall energy resolution of $7 \%$.

There are $78 v_{\mu}$ candidate events in the far detector selected by this process. If there were no oscillations, a projection of the flux seen in the near detector would result in $473 \pm 30$ such events. Of those 78 events, it is estimated that there are backgrounds of 3.4 neutral current, $0.23 v_{e}, 0.27 v_{\tau}$, and 2.7 cosmic-ray-induced events (the blue line in Fig. 2's spectrum). Neutrino oscillations are fit to the resulting spectra, producing a best fit (the red line) of $\Delta m_{32}^{2}=(+2.67 \pm 0.11) \times 10^{-3} \mathrm{eV}^{2}$ and $\sin ^{2} \theta_{23}$ at the two statistically degenerate values $0.404_{-0.022}^{+0.030}$ and $0.624_{-0.030}^{+0.022}$ (at $68 \%$ c.l.) in the normal hierarchy. For the inverted hierarchy, $\Delta m_{32}^{2}=(-2.72 \pm 0.11) \times 10^{-3} \mathrm{eV}^{2}$ and $\sin ^{2} \theta_{23}=$ 

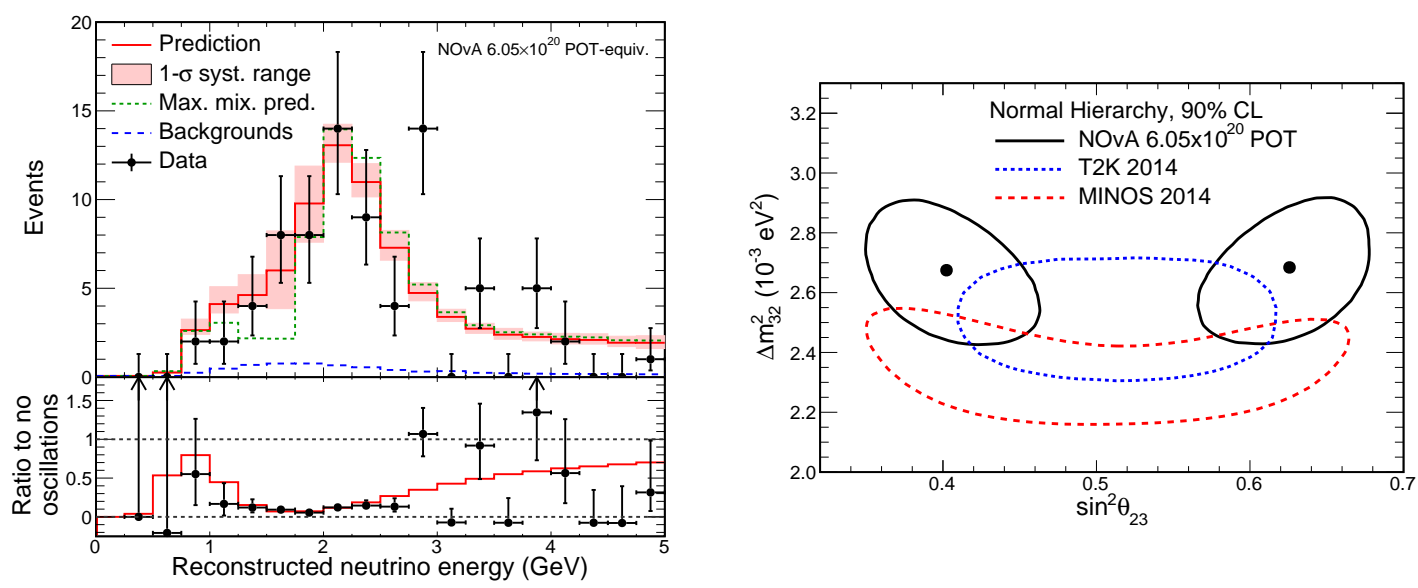

Figure 2: Left: A reconstructed $v_{\mu}$ energy spectrum of data in the nova far detector (black dots with statistical errors) compared to the best oscillation fit prediction with systematic errors (red). Backgrounds are the small blue dashed line, and the prediction at maximal mixing the green dashed line. The ratio of observed data to the unoscillated $v_{\mu}$ spectrum derived from the near detector is the bottom part of the graph. Right: $90 \%$ allowed contours on the oscillation parameters: these results are the black lines, other experiments the dashed lines. Note the separation of the NOvA data from $\sin ^{2} \theta_{23}=0.5$. Plots from [4].

$0.398_{-0.022}^{+0.030}$ or $0.618_{-0.030}^{+0.022}$ (again at $68 \%$ c.l.). This analysis is mostly insensitive to different values of $\Delta_{C P}$, so potential variations therein have been taken as a systematic error. The resulting allowed region is shown on the right of Fig. 2.

Of particular interest is the fact that if $\sin ^{2} \theta_{23}=0.5$ ("maximal mixing"), then there are too many $v_{\mu}$ surviving at this $\mathrm{L} / \mathrm{E}$ corresponding to the first oscillation minimum, even more than can be accounted for by background events or by the smearing of bins by the detector's energy resolution. This is shown by the difference between green and red lines in the spectrum, and results in the separation of the two $90 \%$ allowed regions in the right of Fig. 2 not approaching the middle $\left(\sin ^{2} \theta_{23}=0.5\right.$ ) of the plot. Maximal mixing is disfavored by the data at $2.6 \sigma$. For a full discussion of the details of the fitting and systematic errors please see [4].

\section{Electron Neutrino Appearance}

While the dominant oscillation effect at this energy and baseline is the muon neutrino disappearance discussed above, recent work by many experiments (summarized in [6]) has shown that some $v_{\mu}$ appear as $v_{e}$, governed by the mixing angle $\theta_{13}$. NOvA was designed to measure this process, and thus has some sensitivity to sub-dominant oscillation terms involving the neutrino mass hierarchy, CP-violating phase $\delta_{C P}$, and the octant of $\theta_{23}$. Counting the $v_{e}$-like events and separating them from the other two categories shown in Fig. 1 is handled by a Convolutional Visual Network $(\mathrm{CVN})$ as discussed in [11]. This improves the sensitivity of the result by $30 \%$ over traditional reconstruction algorithms, and results in $73.5 \%$ efficiency and $75.5 \%$ purity of $v_{e}$ events in the final sample (as determined from Monte Carlo studies), while rejecting $97.6 \%$ of the neutral current and $99.0 \%$ of the $v_{\mu}$ charged current beam backgrounds. Thousands of $v_{e}$ candidates are selected in high-flux present at the near detector, where the baseline is too short for any oscillation signal to be 
present. This results in a map of the backgrounds to be expected at the far detector (see the left part of Fig. 3). After reconstruction, $33 v_{e}$ candidates are found at the far detector, of which $8.2 \pm 0.8$ correspond to the expected background in the absence of $v_{e}$ appearance oscillations (the right part of Fig. 3). For complete details see [5].
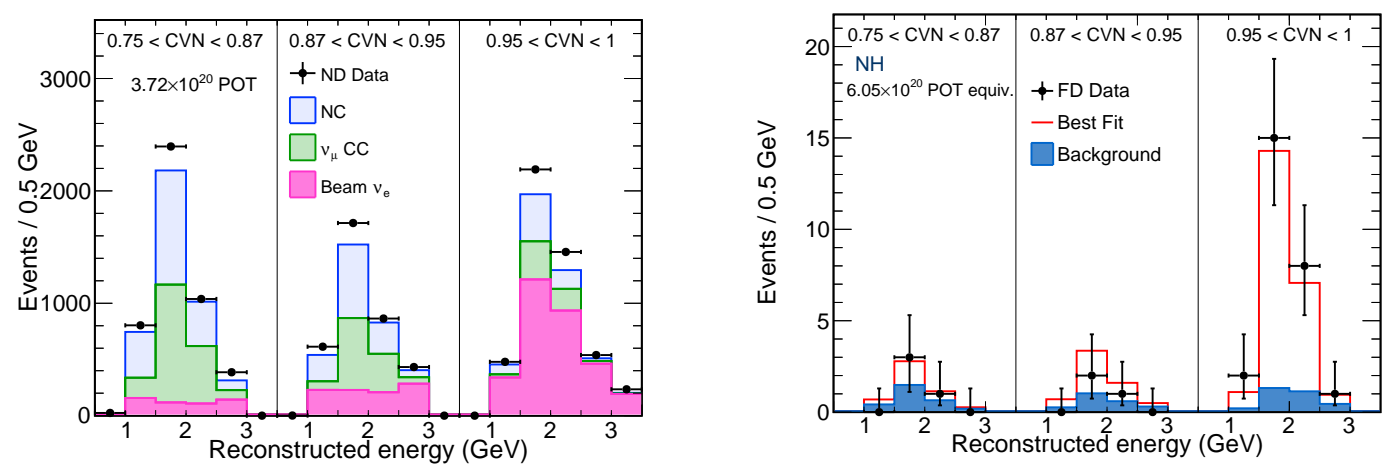

Figure 3: Left: Reconstructed $v_{e}$ candidates energy spectrum of data in the nova near detector (black dots with statistical errors) compared to estimated sources of backgrounds for three different ranges of signal purity. No oscillations are present at the short baseline of the near detector, all selected events are thus backgrounds. Right: The same for the far detector, with all backgrounds (as extrapolated from the near detector data) lumped together, showing the surplus of $v_{e}$-like events caused by appearance oscillations. Plots from [5].

To obtain oscillation parameters, these data are fit simultaneously with the $v_{\mu}$ data discussed previously, taking the precise value of $\theta_{13}$ and its uncertainty from reactor experiments [6] as a given. The inverted mass hierarchy predicts too few $v_{e}$ events appearing at the far detector (see the left part of Fig. 4), so if $\theta_{23}$ is in the lower octant, is disfavored at all values of $\delta_{C P}$ by at least 93\% c.l. Other combinations of hierarchy and octant are more consistent with the data (see the right half of Fig. 4).
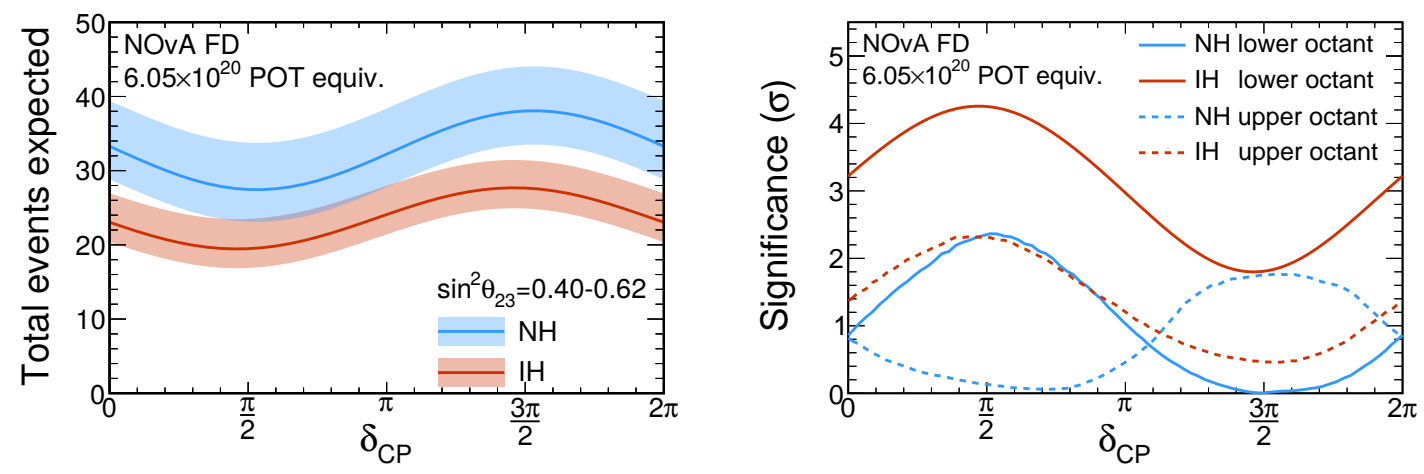

Figure 4: Left: The number of $v_{e}$ candidates expected at the NOvA far detector as a function of $\delta_{C P}$ for the normal (blue) and inverted (red) mass hierarchies. The shaded bands correspond to the uncertainty on $\theta_{23}$. Right: The significance at which each of the four possible combinations of mass hierarchy and $\theta_{23}$ octant are disfavored as a function of $\delta_{C P}$. Plots from [5]. 


\section{Summary}

The NOvA experiment observes both $v_{\mu}$ disappearance and $v_{e}$ appearance oscillations in the primarily $v_{\mu}$ NuMI beam. Analysis of both these modes using the first $6.05 \times 10^{20}$ protons on target exposure have helped refine our knowledge of oscillation parameters. Muon neutrino disappearance points to non-maximal mixing with $2.6 \sigma$ significance and best-fit oscillation parameters of of $\Delta m_{32}^{2}=(+2.67 \pm 0.11) \times 10^{-3} \mathrm{eV}^{2}$ and $\sin ^{2} \theta_{23}$ at the two statistically degenerate values $0.404_{-0.022}^{+0.030}$ and $0.624_{-0.030}^{+0.022}$ (at $68 \%$ c.l.) in the normal hierarchy. Electron neutrino appearance oscillations allow the testing of the four possible permutations of $\theta_{23}$ octants mass hierarchies as a function of $\delta_{C P}$ given the $\theta_{13}$ constraints from reactor experiments and NOvA's own $\theta_{23}$ measurements, disfavoring the lower $\theta_{23}$ octant by at least $93 \%$ c.l. at all values of $\delta_{C P}$ in the case of the inverted hierarchy.

A comparison of an exposure of $9 \times 10^{20}$ pot neutrino exposure (this data is in the can) with a similar amount of anti-neutrino data (the data being taken since 2016) is planned for the summer of 2018.

\section{References}

[1] D. S. Ayres et al. [NOvA Collaboration], The NOvA Technical Design Report, FERMILAB-DESIGN-2007-01.

[2] K. Anderson et al., The NuMI Facility Technical Design Report, FERMILAB-DESIGN-1998-01.

[3] P. Adamson et al. [MINOS Collaboration], The NuMI Neutrino Beam, Nucl. Instrum. Meth. A 806, 279 (2016) [arXiv:1507.06690 [physics.acc-ph]].

[4] P. Adamson et al. [NOvA Collaboration], Measurement of the neutrino mixing angle $\theta_{23}$ in NOvA, Phys. Rev. Lett. 118, no. 15, 151802 (2017) [arXiv:1701.05891 [hep-ex]].

[5] P. Adamson et al. [NOvA Collaboration], Constraints on Oscillation Parameters from $v_{e}$ Appearance and $v_{\mu}$ Disappearance in NOvA, Phys. Rev. Lett. 118, no. 23, 231801 (2017) [arXiv:1703.03328 [hep-ex]].

[6] C. Patrignani et al. [Particle Data Group], Review of Particle Physics, Chin. Phys. C 40, no. 10, 100001 (2016).

[7] K. Abe et al. [T2K Collaboration], Measurements of neutrino oscillation in appearance and disappearance channels by the T2K experiment with $6.60^{20}$ protons on target, Phys. Rev. D 91, no. 7 , 072010 (2015) [arXi;5A;5Av:1502.01550 [hep-ex]].

[8] A. Norman et al., Performance of the NOvA Data Acquisition and Trigger Systems for the full $14 \mathrm{kT}$ Far Detector, J. Phys. Conf. Ser. 664, 082041 (2015).

[9] A. Sheshuko, Detection of the galactic supernova neutrino signal in the NOvA experiment, in proceedings of ICRC 2017 (this conference).

[10] A. Habig, S. Tognini, R. Gomes, M.Goodman, and P.Schreiner, Seasonal Variations of Multiple Muon Events in MINOS and NOvA, in proceedings of ICRC 2017 (this conference).

[11] A. Aurisano et al., A Convolutional Neural Network Neutrino Event Classifier, JINST 11, no. 09, P09001 (2016) [arXiv:1604.01444 [hep-ex]]. 